\title{
TRADUCCIÓN DEL CONOCIMIENTO: PAUTAS BÁSICAS PARA LOS PROFESIONALES DE LA SALUD PÚBLICA
}

\author{
Nina Van Der Stuyft ${ }^{1, a}$, Alonso Soto ${ }^{2,3, b}$, Lely Solari'2,C
}

\begin{abstract}
RESUMEN
La traducción del conocimiento (TC) en Salud Pública involucra el uso de la evidencia científica por parte de los tomadores de decisiones al momento de generar políticas sanitarias, con el fin de acelerar los beneficios resultantes de la investigación e innovación en el fortalecimiento de los sistemas de salud y la salud de la población. En este documento se ofrecen las pautas básicas, como son las definiciones y marcos conceptuales relacionados la sistematización de los procesos de TC, para una mejor comprensión del tema por parte de los profesionales de la salud cuyas funciones están orientadas a la salud pública. Las diferentes aproximaciones a la TC comparten, en esencia, que ella está conformada por dos elementos básicos: un producto (información) desarrollado mediante el método científico con un formato amigable para su público objetivo, y una acción destinada a sustentar o promover una práctica relacionada a la salud utilizando el producto descrito. La comprensión y aplicación de la TC es un elemento clave para la utilización eficaz del conocimiento en la toma de decisiones.
\end{abstract}

Palabras clave: Traducción del conocimiento; Toma de decisiones; Salud Pública (fuente: DeCS BIREME).

\section{KNOWLEDGE TRANSLATION: THE BASICS FOR PUBLIC HEALTH PROFESSIONALS}

\begin{abstract}
Knowledge translation (KT) in Public Health involves the use of scientific evidence by decision makers when generating health policies directed to accelerate the benefits resulting from research and innovation in health system and ultimately people's health. In this paper we review the definitions and conceptual frameworks related to systematization of KT processes. The different approaches to KT share the concept that KT is composed by two main elements: a product (information) developed through scientific method with a friendly format for the target public and an action devoted to support or promote a health related practice using the aforementioned product. The understanding and application of KT is a key element for effective use of knowledge in decision making.
\end{abstract}

Key words: Knowledge translation; Decision making; Public health (source: MeSH NLM).

\section{INTRODUCCIÓN}

El uso de evidencia científica procedente de estudios de investigación para fundamentar las políticas sanitarias y prácticas en salud pública es un tema de creciente interés a nivel global (1). Sin embargo, en la mayoría de los sistemas de salud de países en vías de desarrollo, e incluso en muchos países desarrollados, no necesariamente existen mecanismos que favorezcan estas prácticas ${ }^{(2)}$. Es más, en muchos de estos sistemas de salud existen elementos que potencialmente pueden distanciar la información científica de las políticas sanitarias y que van desde la percepción de la falta de pragmatismo de la información científica por parte de los decisores hasta sistemas legislativos burocráticos. Es por eso de suma importancia que la comunidad ligada al campo de la salud pública, en especial los profesionales de esta disciplina y la sociedad en general conozca el potencial impacto del uso de la evidencia científica en la toma de decisiones y con suerte, se convierta en promotora de esta iniciativa.

En este proceso existe un concepto clave que debe conocerse y es el de "traducción del conocimiento". Es conveniente en este contexto conocer su lógica, cómo se concibe dentro los sistemas de salud, cuáles son los actores clave que deberían de abordar el tema y qué elementos podrían funcionar como potenciales

\footnotetext{
University of Ghent. Bélgica.

Escuela de Medicina. Universidad Peruana de Ciencias Aplicadas. Lima, Perú.

Departamento de Medicina. Hospital Nacional Hipólito Unanue. Lima, Perú.

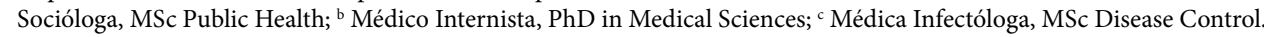

Recibido: 28/10/2015 Aprobado: 06/07/2016
} 
facilitadores u obstáculos en su desarrollo. El presente documento tiene como objetivo brindar una revisión de las principales teorías asociadas a traducción del conocimiento para su mejor comprensión, esperando con esto contribuir a la difusión y aplicación de este elemento en nuestra región y fomentar el debate y la investigación en el tema. Si bien algunas de estas teorías fueron desarrolladas hace décadas por las ciencias sociales, en la actualidad siguen vigentes y aplicables al ámbito de la salud.

\section{DEFINICIONES}

En la literatura internacional hay varios términos que se utilizan para describir la transformación del conocimiento científico en propuestas de acción ${ }^{(3,4)}$ y los países la asumen desde distintos enfoques. Por ejemplo, la literatura norteamericana usa más comúnmente las palabras "diseminación" y "difusión", mientras que investigadores canadienses prefieren usar términos como "transferencia", "intercambio" y "traducción" en sí del conocimiento. ${ }^{5}$ La diversidad en la terminología está en parte explicada por la diversidad de contextos y actores en los que el proceso se da en los distintos países. Algunos reportes simplemente describen la relación entre el investigador y el usuario de las evidencias y otros describen un proceso ecológico, con un gran número de actores.

En una de las revisiones sistemáticas más importantes del tema, Tetroe et al. ${ }^{(6)}$ encontró 29 términos diferentes para definir algún aspecto de este proceso. Lo que la mayoría de las definiciones tienen en común es la referencia a acciones que contribuyen a disminuir la brecha entre el conocimiento derivado de investigación y la implementación de este conocimiento por tomadores de decisiones con el fin de mejorar los resultados en salud pública ${ }^{(4,7)}$.

En el 2000, el Instituto Canadiense de Investigación en Salud (Canadian Institute for Health Research [CIHR]), introdujo específicamente el término "traducción del conocimiento" (TC), el cual es el más utilizado en la actualidad ${ }^{(8)}$. El CIHR define TC como: El intercambio, la síntesis y la aplicación éticamente sólida de conocimientos -dentro de un conjunto complejo de interacciones entre investigadores y usuarios- para acelerar la captación de los beneficios de investigación para los canadienses a fin de lograr una mejor salud, servicios y productos más eficaces y un sistema de salud fortalecido ${ }^{(9)}$.

Desde entonces, otras organizaciones han sugerido adaptaciones de esta definición (7). Por ejemplo, la Organización Mundial de Salud ha definido la TC como: La sintesis, el intercambio y la aplicación del conocimiento por utilizadores pertinentes para acelerar los beneficios de innovaciones globales y locales en el fortalecimiento de los sistemas de salud y en mejorar la salud de las personas ${ }^{(10)}$.

El CIHR indica que la principal característica de la TC es que incluye todas las etapas desde la creación de conocimientos nuevos hasta la aplicación de estos para beneficiar a la sociedad ${ }^{(7)}$. Las características de la TC según el CIHR se presentan en la Tabla 1.

Es pertinente aclarar que cuando decimos "investigadores" nos referimos a personas que manejan el método científico y desarrollan el diseño, ejecución, análisis y diseminación de los resultados del estudio de investigación, mientras que por otro lado los "tomadores de decisiones" (utilizadores de evidencia) son las personas que tienen responsabilidad en la selección y/o aplicación de intervenciones de salud y esto puede darse a varios niveles: pueden ser directores de hospitales/instituciones/organizaciones o personas con cargos políticos como jefes de programas de control y gerentes en los ministerios.

\section{TEORÍAS RELACIONADAS A TRADUCCIÓN DEL CONOCIMIENTO}

A continuación, se analizan algunas de las teorías clave sobre TC que se encuentran en la literatura científica. Para la indagación bibliográfica, se buscó un encabezado de tema médico (término Mesh) para traducción del conocimiento en la base de datos MEDLINE. Sin embargo, sorprendentemente, solo existe un término para investigación traslacional, que no es el mismo concepto. Por esto se buscó en la base de datos MEDLINE con la estrategia "traducción del conocimiento" $Y$ "conceptos", con fecha 31 de diciembre de 2015. Solo se incluyeron en la presente revisión las publicaciones que incluyeran teorías o modelos explicativos de TC, en general, es decir, que no fueran

Tabla 1. Características de la traducción del conocimiento según el Centro Canadiense de investigación para la Salud

Incluye todas las fases entre la creación de conocimientos
nuevos y la aplicación de estos conocimientos.
Necesita comunicación multidireccional.
El proceso es interactivo
Requiere colaboraciones entre los actores pertinentes.
El proceso no es lineal.
Enfatiza en el uso de conocimientos basados en
investigación. Sin embargo, esto puede ser usado en
conjunción con otros tipos de conocimiento.
Incluye múltiples actividades.

Adaptado de NCDDR, $2005^{(8)}$ 
específicas para un tema de salud en particular, como cáncer; enfermedades crónicas u otros. Cabe notar que la mayoría de los modelos explicativos incluidos fueron encontrados en la búsqueda secundaria, es decir, en la revisión de la bibliografía de los artículos seleccionados. Asimismo, se complementó la búsqueda con Google Scholar para identificar libros relevantes sobre el tema.

\section{Enfoque basado en el estudio de la utilización del conocimiento}

Uno de los primeros investigadores que intentó explicar el concepto de 'utilización de evidencias' fue Carol Weiss, ${ }^{(12,13)}$ quien definió seis tipos de significados diferentes que los autores dan a la utilización de evidencias en políticas públicas ${ }^{(12)}$. A pesar de que estos se basan en investigación social, consideramos que los conceptos también son aplicables a la investigación en salud.

\section{Modelo de utilización impulsada por el conocimiento}

La definición de utilización de evidencias en este modelo se basa en las ciencias naturales. Según este modelo, hallazgos de investigación llevan directamente al uso de los resultados en políticas. Se supone que los eventos pasan por la siguiente secuencia: investigación básica --> investigación aplicada --> desarrollo --> aplicación. La investigación aplicada tiene como meta la confirmación de la investigación básica. Luego, se desarrollan estrategias para la implementación de los resultados, los cuales son aplicados en la última fase ${ }^{(12)}$.

\section{Modelo de resolución de problemas}

Según este modelo de utilización, las conclusiones y evidencias derivadas de la investigación ayudan a solucionar un problema. Este problema es definido por el utilizador del conocimiento, quien emplea la investigación para proveer respuestas o claves que faltan para la solución del problema. El principal papel de la investigación es ayudar a seleccionar medios apropiados para cumplir con las metas políticas.

Según este modelo, el conocimiento científico puede influir en la política de dos maneras. La investigación puede ser realizada antes de que el problema sea definido, y ser usada cuando sea necesaria. En este caso, los tomadores de decisiones buscan la información que necesitan en investigaciones ya hechas. Otro camino de influencia es que los utilizadores de la evidencia necesitan una cierta información y reclutan a investigadores para levantar datos. El proceso es el graficado en la Figura 1.

\section{Modelo interactivo}

Este significado de la traducción de evidencias es más amplio: el conocimiento científico puede influir en las decisiones por diferentes caminos, debido a que los tomadores de decisiones no solamente usan investigaciones como fuentes de información. En contraste con los dos primeros modelos, este no es lineal. El proceso de toma de decisiones es un conjunto de conexiones que forman un esquema complicado. Los investigadores son simplemente un grupo de actores entre varios otros. Ejemplos de otros actores son periodistas, políticos, grupos de interés e incluso amigos, entre otros.

\section{Modelo político}

Esta definición parte del principio que el conjunto de intereses que tienen los políticos definen las opiniones de los tomadores de decisiones. En este caso, no es probable que la investigación nueva sea utilizada de manera prioritaria. Sin embargo, sí es usada para avalar las posiciones o las decisiones de los utilizadores. En este contexto, se tiene que estar alerta a la mala interpretación o distorsión de los resultados. Una condición para que este modelo sea aplicable es la igualdad en el acceso a la información por cada actor.

\section{Modelo táctico}

En esta forma de utilización de evidencias las investigaciones no son utilizadas por su contenido, sino como una táctica política. Por ejemplo, los tomadores de decisiones usan el argumento de que 'hay una investigación en curso' para no tener que hacer frente a problemas. Otra posibilidad es que justifican acciones criticadas con el hecho que "son basadas en evidencias científicas" y así evitan tomar la responsabilidad para resultados políticos que son poco populares.

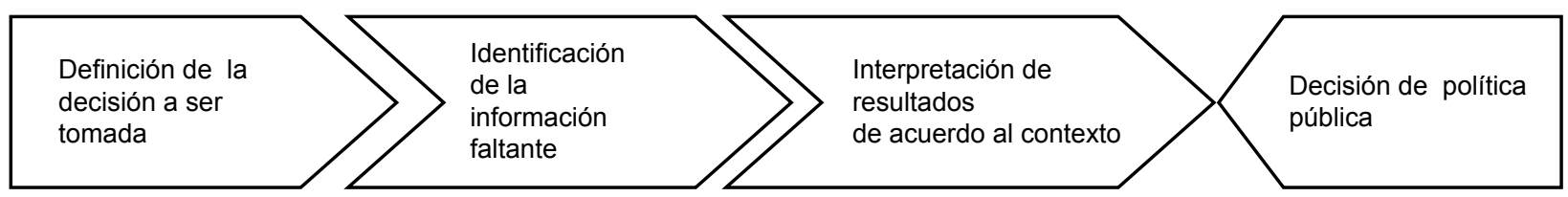

Adaptado de Weiss, $1979^{(12)}$

Figura 1 . Pasos del modelo de resolución de problemas 


\section{Modelo de esclarecimiento}

En este modelo, es más bien el conjunto de teorías y conceptos generales de las ciencias sociales los que influyen en las políticas en lugar de estudios aislados. Los utilizadores de los conocimientos no se pueden referir a un estudio específico para justificar sus decisiones, pero tienen la impresión de que las ciencias sociales les han dado antecedentes en los cuales basar sus decisiones.

Modelo de Investigación como parte de 'la empresa intelectual de la sociedad'

En esta imagen de utilización de evidencias, hay mucha interacción entre la política y las ciencias. Cuando hay un interés político acerca de un cierto tema, el financiamiento para estudios acerca de este tema es mayor, lo que significa que los investigadores están más interesados en estudiarlo.

La separación de varios significados de la traducción de conocimientos de CarolWeiss ${ }^{(12)}$ es muyútil para clasificar la literatura existente, y comprender cómo funcionan los sistemas de algunos países latinoamericanos, pero se limita a un ejercicio descriptivo: casi no se brindan orientaciones concretas a investigadores para mejorar el proceso o los pasos concretos a tomar para facilitar el uso de evidencia científica ${ }^{(15)}$.

\section{Enfoque basado en los actores involucrados: teoría de las dos comunidades}

La asunción clave en esta teoría es que los investigadores y los tomadores de decisiones son dos grupos completamente distintos con diferentes opiniones y percepciones acerca de la investigación ${ }^{(4)}$. El bajo uso de evidencias científicas se explica por las diferencias 'culturales' entre los "productores" y los "utilizadores" de evidencias. Estas diferencias resultan en lo que Caplan ${ }^{(11)}$ define como la "brecha cultural": Los científicos y los tomadores de decisiones viven en mundos completamente diferentes. Los investigadores se enfocan en la ciencia 'pura', en cuanto los utilizadores de evidencias piensan de un modo más práctico y en soluciones inmediatas. El autor sugiere que esta brecha debería ser cerrada mediante un reforzamiento de las interacciones entre ambos grupos ${ }^{(11)}$.

Esta teoría de las dos comunidades puede ser importante para aportar a la comprensión de la problemática, pero resulta un poco estrecha para explicar todas sus dimensiones. Esta teoría supone que solamente hay dos grupos (investigadores y tomadores de decisiones) que tienen un impacto en el uso de evidencias y no tiene en cuenta la existencia de otros factores ni elementos facilitadores y obstáculos propios del sistema.

\section{Enfoque basado en el proceso del conocimiento hasta la acción}

Graham et al. ${ }^{(2)}$ diseñaron un modelo que incluye a un gran rango de actores. Diferentes fases del ciclo de traducción de evidencias pueden ser logradas por estos diferentes actores. Además, dentro de este modelo, la información no se deriva solamente de la investigación científica, sino también de otras formas de conocimiento, como la experiencia. El modelo, a diferencia de los previos, no es solo explicativo sino que fue diseñado para facilitar el uso del conocimiento derivado de la investigación ${ }^{(7)}$.

El proceso de traducción de evidencias según este grupo está dividido en dos submodelos: creación de conocimiento y acción.

\section{Creación de conocimientos}

La creación de conocimiento consiste de tres fases: consulta del conocimiento, síntesis del conocimiento y herramientas y productos del conocimiento, y es visualizado como un embudo. Cuanto más refinado y más útil para los usuarios, el conocimiento se encuentra mejor posicionado.

La fase de la consulta del conocimiento representa la totalidad de estudios o información disponible acerca de un cierto tema. La calidad de esta información varía y es bastante difícil de procesar de una manera eficiente.

La segunda fase se llama 'síntesis del conocimiento' y representa la combinación de la información en forma de revisiones sistemáticas, metaanálisis o metasíntesis. La última capa del embudo consiste en las herramientas y los productos del conocimiento. Tenemos como ejemplos a las guías de práctica, reglas y ejemplos de herramientas. Todos estos están listos para ser utilizados. Son claros y en general proveen recomendaciones explicitas.

\section{Ciclo de acción}

La fase de acción es representada por un círculo que contiene las actividades y los procesos necesarios para la aplicación de conocimientos. Cada etapa en este ciclo puede ser influenciada por otra etapa y por la fase de creación del conocimiento.

La primera etapa es la identificación del problema y la búsqueda de información acerca de este problema. Luego se debe adaptar el conocimiento disponible al contexto en el que va a ser aplicado. En la siguiente fase, posibles barreras que pueden impedir el uso de evidencias son identificadas. 
Seguidamente, las intervenciones para promover la implementación del conocimiento son diseñadas y realizadas. Luego, la utilización del conocimiento tiene que ser supervisada. Para finalizar, el impacto del uso del conocimiento debe de ser evaluado y el sostenimiento de su uso debe de ser asegurado.

Este modelo es muy útil para una comprensión completa del proceso de traducción del conocimiento en acción, pero debe de ser asumido de acuerdo a cada contexto.

\section{Enfoque basado en la búsqueda de una traducción eficaz del conocimiento: Las preguntas de Lavis}

Otro ejemplo de un modelo bastante aplicable para investigadores es el modelo de Lavis et al. ${ }^{(14)}$. Este modelo está basado en una revisión sistemática de la literatura y distingue cuatro públicos objetivos: destinatarios de servicios (pacientes y ciudadanos), proveedores de servicios (clínicos), tomadores de decisiones de gestión (gerentes de hospitales y organizaciones comunitarias) y tomadores de decisiones a nivel local, provincial o estatal.

Además, este modelo está estructurada por cinco preguntas las cuales los investigadores deben preguntarse a sí mismos para asegurar una traducción de evidencias más eficiente: ¿qué?, ¿quién?, ¿por quién?, ¿cómo? y ¿con qué objeto? ${ }^{(12)}$.

¿Qué información debería de ser traducida (transferida) a los tomadores de decisiones?

Los investigadores deben transferir datos específicos en los cuales se pueden basar acciones, en lugar de un reporte o los resultados de una única investigación. Muchas veces los resultados no son concluyentes o aplicables y no proponen soluciones para problemas concretos.

\section{¿A quién debería ser transferido el conocimiento?}

El público objetivo debe ser bien identificado y definido, y la estrategia de traducción de la evidencia debe de ser adaptada a este público, al tipo de decisiones que tienen que hacer y al ambiente en los cuales se tienen que tomar las decisiones.

\section{¿Por quién debería ser transferido el conocimiento?}

La credibilidad de quien transfiere la información tiene un papel importante en el proceso de traducir la evidencia práctica. Organizaciones y profesionales del estado tienen más credibilidad y son fuentes de información para los tomadores de decisiones.

\section{¿Cómo debería ser transferido el conocimiento?}

Los resultados de investigaciones deben ser presentados de manera interactiva entre investigador $y$ tomador de decisiones. Cuando la evidencia es presentada de modo pasivo, es decir publicando solamente resultados generales, la probabilidad de que sea utilizada es baja.

¿Con qué objeto debería ser transferido el conocimiento?

Los investigadores deberían definir muy bien sus objetivos y los efectos deseados. De este modo, es más fácil adaptarlos al público al que se dirigen ${ }^{(16)}$.

\section{El modelo de Jacobson y Butteril}

Otro ejemplo de un modelo aplicable por investigadores es el sugerido por Jacobson et al. (17) quienes presentan una secuencia de preguntas que los investigadores tienen que proponerse ${ }^{(8)}$ con la finalidad de conocer mejor al grupo de usuarios del conocimiento ${ }^{(14)}$.

El modelo contiene cinco elementos: el grupo de utilizadores, la investigación, el tema, la relación entre el usuario y el investigador y las estrategias de diseminación. Para cada uno de estos elementos, los investigadores han formulado una serie de preguntas para que el investigador pueda organizar su información ${ }^{(17)}$

Además de los enfoques revisados, se han definido un gran rango de factores que facilitan u obstaculizan el uso de los resultados de investigación. Ejemplos de elementos facilitadores son la inclusión de tomadores de decisiones en el proceso de investigación, sobre todo de la formulación de la misma, la relevancia y calidad de la investigación y la inclusión de datos sobre efectividad de los resultados según sus necesidades, entre otros. Otro aspecto de suma relevancia es el contacto personal entre el investigador y el responsable de la política ${ }^{(1,19,21)}$. Por otro lado, ejemplos de factores que impiden el uso de evidencia son: ingenuidad política de los investigadores, déficit de tiempo, falta de acceso a la literatura, escasez de recursos, resistencia al cambio, enfoque en la validez de la evidencia en lugar de en su aplicabilidad, entre otros. Se debe de tomar en cuenta que, en general, las políticas son afectadas por más factores que son ajenos a la investigación ${ }^{(13)}$. Ejemplos de estos son la estrategia y presión de los grupos de interés, la opinión pública ${ }^{(18,21)}$.

\section{DISCUSIÓN}

Si hay algo que tienen en común todas estas aproximaciones al tema de traducción de conocimiento son los dos elementos básicos que lo conforman: en primer lugar un producto (información) desarrollado mediante el método científico, con un formato amigable para su público objetivo y, por otro lado, una acción destinada a sustentar o promover una práctica relacionada a la salud utilizando el producto descrito. Por fuera de este núcleo base, los modelos conceptuales varían entre sí de acuerdo a varios factores, entre los cuales podemos incluir a los actores (desde los 
modelos "simples", que únicamente consideran a los investigadores y los tomadores de decisiones, hasta los modelos más realistas que consideran un mayor espectro de actores y las interacciones complejas que hay entre ellos) y las motivaciones o "fuerzas motrices" que impulsan a estos grupos, desde los modelos en los que es el investigador el que promueve el uso de la información, hasta aquellos en los que son los tomadores de decisiones quienes la solicitan.

Más que el lector se incline por alguna de estas aproximaciones, nuestro objetivo es que conozca estos modelos explicativos y sus principales elementos formuladores para que pueda aplicarlos en la medida de lo posible en su práctica sanitaria, y a su vez difundirlos entre su entorno, para que cada vez más personas sepan de la importancia de la traducción del conocimiento.

Desde nuestro punto de vista, en estos modelos hay una subestimación generalizada del usuario final, que es la comunidad. La mayoría están centrados en la interacción entre los investigadores y tomadores de decisiones y las fuerzas push y pull, pero sin enfatizar de manera suficiente el hecho del que son los ciudadanos en general los que deberían ser los más interesados en hacer la incidencia para que las políticas públicas que los afectan estén basadas en evidencia científica. Así como la sociedad civil se organiza en temas que les interesan, como los derechos reproductivos y otros, sería ideal que hayan colectivos y organizaciones civiles que impulsen este tema. Haciendo un paralelo, aunque un poco lejano, la investigación clínica ya está nutriéndose de la corriente de shared decision making en la que clínicos y pacientes comparten la mejor evidencia disponible al momento de tomar decisiones ${ }^{(22)}$, y es apoyada por grupo de la comunidad civil. Por ejemplo, en Salud Pública sería interesante utilizar una aproximación similar con las comunidades que resultan afectadas por acciones de salud pública que se basan en evidencia científica y cuyo real impacto muchas veces no se cuantifica adecuadamente, como por ejemplo poblaciones afectadas por enfermedades crónicas, etc.

Independientemente de cual sea la realidad de cada país latinoamericano, consideramos que es muy importante hacer un esfuerzo por conocer a fondo su modelo existente (o inexistente) de TC, hacer lo necesario para estandarizar la TC mediante una legislación pertinente, evaluar los mecanismos que puedan acercar a los dos actores más importantes sin menospreciar al resto $y$, sobre todo, transparentar los procesos de toma de decisiones en salud pública y volverlos susceptibles de las rendiciones de cuentas correspondientes.

Para esto, es fundamental desarrollar investigación primaria sobre este tema y esperamos que esta revisión justamente brinde insumos para que investigadores latinoamericanos se interesen en hacer estudios referentes a TC. En la Tabla 2 proponemos algunas
Tabla 2. Líneas de investigación propuestas para Traducción del Conocimiento

\begin{tabular}{ll}
\hline Aspecto a abordar & \multicolumn{1}{c}{ Especificaciones } \\
\hline $\begin{array}{l}\text { Impacto del uso de } \\
\text { evidencia científica en } \\
\text { la toma de decisión }\end{array}$ & $\begin{array}{l}\text { Medición del impacto (resultados de } \\
\text { salud) del uso de herramientas de } \\
\text { Traducción de Conocimiento y las } \\
\text { consecuentes decisiones en salud } \\
\text { pública } \\
\text { De qué maneras particulares } \\
\text { prefieren los decisores que se les } \\
\text { presente la evidencia científica, } \\
\text { cómo podrían aceptar cambiar sus } \\
\text { fuentes de información, etc. }\end{array}$ \\
$\begin{array}{l}\text { Preferencias de } \\
\text { los tomadores de } \\
\text { decisiones }\end{array}$ & $\begin{array}{l}\text { Uso de las redes sociales en la } \\
\text { concientización de la comunidad } \\
\text { sobre uso de la evidencia científica } \\
\text { para salud pública, impacto de } \\
\text { los diálogos públicos para el } \\
\text { delineamiento de las políticas, etc. }\end{array}$ \\
\hline $\begin{array}{l}\text { Herramientas para } \\
\text { incorporar a la } \\
\text { comunidad en la toma } \\
\text { de decisiones en salud } \\
\text { pública }\end{array}$ &
\end{tabular}

líneas de investigación a desarrollar en base a los vacíos que nosotros hemos observado en esta revisión.

Los modelos expuestos en este trabajo nos muestran que hay una gran diversidad de enfoques referentes a TC en la literatura científica. El futuro de la TC debe ir orientado a evaluar en "el campo" estrategias que mejoren la incorporación de los resultados de estudios de investigación a las políticas públicas, sobre todo desde la perspectiva de los decisores. Otro aspecto importante será la construcción de los procesos e incluso "sistemas" nacionales de traducción del conocimiento. Para esto, no solo será importante la participación de los investigadores y actores involucrados en la traducción del conocimiento y en la adopción del mismo, sino también la voluntad política de las personas que tienen poder de decisión respecto a la estructura y funcionamiento del sistema de salud, quienes deberán de convencerse, como lo estamos nosotros, de la relevancia de estos procesos y de la participación de la comunidad en general.

En la década de los setenta, el Dr. Archibald Cochrane consideraba como uno de los grandes retos para la profesión médica la falta de organización de sumarios de los ensayos clínicos. Nosotros debemos ver como un reto importante para nuestro siglo la creación de sistemas efectivos para informar las políticas públicas con investigación científica de calidad.

Contribuciones de autoría: NVDT y LS han participado en la concepción del artículo, revisión de literatura, redacción y aprobación de la versión final. AST ha participado en la revisión de literatura, redacción del artículo y aprobación de la versión final. Todos los autores han aprobado la versión final del artículo y se hacen responsables del contenido del mismo.

Fuentes de financiamiento: autofinanciado.

Conflictos de interés: los autores declaran no tener conflictos de interés. 


\section{REFERENCIAS BIBLIOGRÁFICAS}

1. Orton L, Lloyd-Williams F, TaylorRobinson D, O'Flaherty M, Capewell $S$. The use of research evidence in public health decision making processes: a systematic review. PLoS One. 2011;6(7):e21704. doi: 10.1371/ journal.pone.0021704.

2. Ritter A. How do drug policy makers access research evidence? Int J Drug Policy. 2009;20(1):70-5. doi: 10.1016/j. drugpo.2007.11.017.

3. Davies HTO, Nutley SM. Learning more about how research-based knowledge gets used: guidance in the development of new empirical research. New York: William T. Grant Foundation; 2008.

4. Graham ID, Logan J, Harrison MB, Straus SE, Tetroe J, Caswell W, et al. Lost in knowledge translation: time for a map? J Contin Educ Health Prof. 2006;26(1):13-24. doi:10.1002/chp.47.

5. Straus SE, Tetroe J, Graham I. Defining knowledge translation. CMAJ. 2009;181(3-4):165-8. doi:10.1503/ cmaj.081229.

6. Tetroe JM, Graham ID, Foy R, Robinson N, Eccles MP, Wensing M, et al. Health research funding agencies' support and promotion of knowledge translation: an international study. Milbank Q. 2008;86(1):125-55. doi:10.1111/j.1468-0009.2007.00515.x.

7. Sudsawad P. Knowledge translation: introduction to models, strategies, and measures. Austin, TX: Southwest Educational Development Laboratory, Nacional Center for the Dissemination of Disability Research; 2007.

8. National Center for Dissemination of Disability Research (NCDDR). Focus. What is knowledge translation? Technical brief N 10 [Internet]. Austin, TX: NCDDR; 2005 [cited 2016 Jul
25]. Disponible en: http://ktdrr.org/ ktlibrary/articles_pubs/ncddrwork/ focus/focus10/Focus10.pdf

9. Canadian Institute of Health Research (CIRH). Knowledge translation strategy 2004-2009 [Internet]. Ottawa: CIRH; 2004 [cited 2015 Oct 18]. Disponible en: http://www.cihr-irsc.gc.ca/e/ documents/kt_strategy_20042009_e.pdf

10. World Health Organization. Bridging the "Know-do" gap: Meeting on knowledge translation in global health, 2005. Geneva: WHO; 2006.

11. Caplan N. The two-communities theory and knowledge utilization. Am Behav Sci. 1979;22(3):459-70. doi:10.1177/000276427902200308.

12. Weiss C. The many meanings of research utilization. Public Adm Rev. 1979;39:426-31.doi:10.2307/3109916.

13. Elliott H, Popay J. How are policy makers using evidence? Models of research utilisation and local NHS policy making. J Epidemiol Community Health. 2000;54(6):461-8.

14. Schryer-Roy AM. Knowledge translation: basic theories, approaches and applications [Internet]. Surrey: IDRC; 2005 [cited 2015 Oct 18]. Disponible en: http://www.fraserhealth.ca/ media/KnowledgeTranslation.pdf

15. Trostle J, Bronfman M, Langer A. How Do Researchers Influence Decision-Makers? Case Studies of Mexican Policies. Health Policy Plan. 1999;14(2):103-14.

16. Lavis JN, Robertson D, Woodside JM, McLeod CB, Abelson J, Knowledge Transfer Study Group. How can research organizations more effectively transfer research knowledge to decision makers? Milbank Q. 2003;81(2):221-48.
17. Jacobson N, Butterill D, Goering P. Development of a framework for knowledge translation: understanding user context. J Health Serv Res Policy. 2003;8(2):94-9. doi:10.1258/135581903321466067.

18. Tseng V. Social Policy Report: The Uses of Research in Policy and Practice. Shar child youth Dev Knowl [Internet]. 2012;26(2):[about 16 p.] Disponible en: http://files.eric.ed.gov/fulltext/ ED536954.pdf

19. Dobbins M, Jack S, Thomas $H$, Kothari A. Public Health DecisionMakers' Informational Needs and Preferences for Receiving Research Evidence. Worldviews Evid Based Nurs. 2007;4(3):156-63. doi:10.1111/j.17416787.2007.00089.x.

20. Dobbins M, Rosenbaum P, Plews N, Law M, Fysh A. Information transfer: what do decision makers want and need from researchers? Implement Sci. 2007;2(1):20.

21. Innvær S, Vist G, Trommald $M$, Oxman A. Health policy-makers' perceptions of their use of evidence: a systematic review. J Health Serv Res Policy. 2002;7(4):239-44. doi:10.1258/135581902320432778.

22. Elwyn G, Laitner S, Coulter A, Walker E, Watson P, Thomson R. Implementing shared decision making in the NHS. BMJ. 2010;341:c5146. doi: 10.1136/bmj.c5146

Correspondencia: Alonso Soto.

Dirección. Escuela de Medicina. Universidad Peruana de Ciencias Aplicadas. Lima, Perú Teléfono: (511) 996128953.

Correo electrónico: sotosolari@gmail.com 